\title{
Statistical Physics of Bose-Einstein Condensation
}

\author{
M. GAJDA ${ }^{a}$ AND K. RZĄżEWSKI ${ }^{b}$ \\ ${ }^{a}$ Institute of Physics, Polish Academy of Sciences \\ al. Lotników 32/46, 02-668 Warsaw, Poland \\ ${ }^{b}$ Center for Theoretical Physics, Polish Academy of Sciences \\ al. Lotników 32/46, 02-668 Warsaw, Poland
}

(Received November 15, 2001)

\begin{abstract}
We review a progress in understanding of statistical properties of a quantum degenerate Bose gas. We show how the Maxwell demon ensemble helps to compute fluctuations of the Bose-Einstein condensate of an ideal Bose gas according to the microcanonical ensemble. Then, we review a method of measuring these fluctuations. Using a soluble model of interacting Bose gas we also stress the importance of higher-order correlation functions. Finally, we review our novel computational method of studying an interacting Bose gas near its critical temperature.
\end{abstract}

PACS numbers: 03.75.Fi, 05.30.Jp

\section{Introduction}

It took seventy years to realize experimentally the Bose-Einstein condensation, a quintessentially quantum phenomenon discovered theoretically in 1924 [1, 2]. It would not happen if not for the progress in cooling and trapping of neutral atoms. The Nobel Committee has recognized its impact in 1997 [3]. The seminal experiments that produced a Bose-Einstein condensate (BEC) in dilute rubidium and sodium gases $[4,5]$, performed in 1995, brought a Nobel Prize to E. Cornell, C. Wieman, and W. Ketterle in 2001.

The discovery has let to an avalanche of papers, both experimental and theoretical, devoted to statistical and dynamical properties of the new quantum state of matter. Even the statistical properties of an ideal Bose gas called for revisiting. 
The textbook theory of Bose-Einstein condensation was developed under standard assumptions of the thermodynamic limit, the spatial homogeneity, and the grand canonical statistical ensemble. In the experiment, the number of atoms is finite and varies between several thousand and several million, far from infinity. The gas is confined by a harmonic potential, so the density changes from point to point. Finally, the system is nearly perfectly decoupled from the environment; in particular, it does not exchange particles with an outside reservoir - the scheme implied by grand canonical ensemble.

In this paper, we shall review the statistical properties of the condensed Bose gas with particular emphasis on our own work. In Sec. 2 we explain on a very simple example the role of Bose symmetry principle in the build-up of the macroscopic population in the non-degenerate ground state of the trapping potential. In Sec. 3 the microcanonical theory of the harmonically, bound ideal Bose gas is sketched. To this end, we review a notion of the Maxwell demon ensemble. In Sec. 4 we review a method of investigating the fluctuations of the condensate. In Sec. 5 the role of higher-order correlation functions is stressed, based on an exactly soluble model of elastically interacting bosons. Finally, in Sec. 6 a novel method of studying the stationary dynamics of the weakly interacting Bose gas based on the high-energy solutions of the time-dependent Gross-Pitaevskii equation is explained.

\section{Why bosons bunch together?}

Quantum particles are indistinguishable. Let us take two states and two particles. There are two ways in which classical particles may be placed in these two states. Two, because classical particles may be labeled. Quantum identical particles have just one way. They cannot be labeled. Among quantum particles, these with an integer spin, bosons, do not mind to be all placed at a single quantum state, while half integer spin particles, fermions, need one state for each. Atoms are build of fermions: electrons, protons, and neutrons. Neutral atoms have as many electrons as protons in the nucleus. Thus the parity of the number of neutrons determines the statistics of the whole atom. This is unusual. Most global, low energy properties of matter depend on the cloud of electrons surrounding the nucleus. Typically only nuclear reactions and the high-energy processes involve the nucleus. Here, at very low temperatures, gros features depend on the composition of the nucleus!

To illustrate the power of indistiguishability let us consider a set of $N$ particles moving in a one-dimensional harmonic binding potential characterized by the angular frequency $\omega$. Let us assume further that the system is described by the canonical ensemble with temperature $T$ as its control parameter. Hence, the density matrix of the system takes a form

$$
\rho_{b, \mathrm{cl}}=\frac{\xi_{i=1}^{N} a_{i}^{+} a_{i}}{Z_{b, \mathrm{cl}}(N, \xi)},
$$


both for bosons and classical particles. $Z_{b, \mathrm{cl}}(N, \xi)$ is the canonical partition function. We have introduced here an abbreviation $\xi=\exp (-\hbar \omega / k T)$, where $k$ is the Boltzmann constant. The physics depends now on the character of the particles. The classical partition function results from the independent summation over all configurations for each particle

$$
Z_{\mathrm{cl}}=\left(\sum_{n=0}^{\infty} \xi^{n}\right)^{N}=\left(\frac{1}{1-\xi}\right)^{N}
$$

which is just the $N$-th power of the single-particle partition function. No correlation is present in the classical ideal gas. Summation for bosons must be restricted. In particular, out of $N$ ! configurations with each particle at different level only one is present for bosons. It is obvious that the indistinguishability is assured if we sum over the restricted range for each index

$$
Z_{b}=\sum_{n_{N}=0}^{\infty} \xi^{n_{N}} \ldots \sum_{n_{2}=n_{3}}^{\infty} \xi^{n_{2}} \sum_{n_{1}=n_{2}}^{\infty} \xi^{n_{1}}=\prod_{n=1}^{N} \frac{1}{1-\xi^{n}} .
$$

Now, for each classical particle, the probability of being in the ground state is $\xi$ and in any place above is remaining $1-\xi$. Therefore, the probability of finding exactly $n$ particles in the ground state out of $N$ is given by

$$
P_{n, \mathrm{cl}}(N, \xi)=\left(\begin{array}{c}
N \\
n
\end{array}\right) \xi^{n}(1-\xi)^{N-n}
$$

The mean number of particles in the ground state and its variance as a function of temperature are shown for $N=1000 \mathrm{in} \mathrm{Fig.} 1$ as dashed lines. This is to be contrasted with the bosonic result, shown with solid lines, which again requires restricted summations

$$
P_{n, b}(N, \xi)=\frac{1}{Z_{b}} \sum_{j_{n}-1}^{\infty} \xi^{j_{n-1}} \ldots \sum_{j_{1}=j_{2}}^{\infty} \xi^{j_{1}}=\xi^{n} \prod_{j=n+1}^{N-n}\left(1-\xi^{j}\right) .
$$

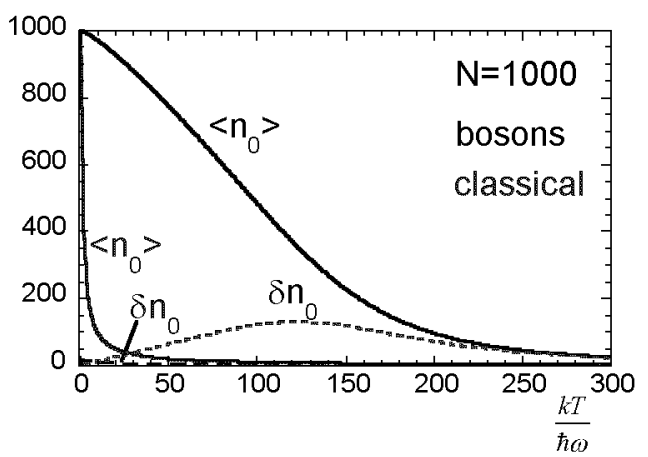

Fig. 1. Temperature dependence of the population of the ground state of $1 \mathrm{D}$ harmonic oscillator and its variance for classical and Bose particles. Note macroscopic population of bosons even for high temperatures. 
The contrast is striking. While both systems occupy the ground state at zero temperature, the macroscopic occupation of this state for bosons occurs already at temperatures, at which there is enough energy to lift all particles to the excited states. All this is solely due to quantum statistics and also takes place in higher number of dimensions.

The reader expecting a rigorous phase transition i.e. a nonanalytic behavior of physical quantities may be disappointed. Two remarks are in order. First: we deal here, as in experiments, with a finite sample. Everything must be smooth. Second: it is a one-dimensional system, so in the absence of (long-range) interactions we do not expect a phase transition, even for $N$ going to infinity.

In the next section we are going to present results for three-dimensional harmonic traps.

\section{The Maxwell demon ensemble}

A standard textbook approach to Bose-Einstein condensation of an ideal Bose gas is based on the grand canonical ensemble [6] introduced by Gibbs. The two control parameters here are the temperature $T$ and the chemical potential $\mu$. The latter determines a mean number of particles in the system. The system is assumed to be in contact with the reservoir of energy, which determines its temperature, and with the reservoir of particles, which determines its chemical potential. A beauty of this ensemble stems from the fact that the grand canonical partition function is explicitly given by the single particle energies $\varepsilon_{i}$ :

$$
\Xi(T, \mu)=\prod_{\varepsilon_{i}} \frac{1}{1-\mathrm{e}^{-\varepsilon_{i} / k T} z},
$$

where the fugacity $z$ is given by

$$
z=\exp (\mu / k T)
$$

Specifying the formula for the three-dimensional isotropic harmonic oscillator, accounting for the degeneracy of its levels, we get

$$
\Xi(\xi, z)=\prod_{n=0}^{\infty}\left[\frac{1}{1-z \xi^{n}}\right]^{\frac{(n+1)(n+2)}{2}}
$$

Useful as a tool to obtain the temperature dependence of the mean number of quanta in the condensed state, the grand canonical ensemble fails when used for the statistics of the condensed fraction. It has long been known [7] that it predicts variance of the occupation of the ground state to be of the order of its mean value even at zero temperature. But in the experiment the number of atoms in a trap is strictly conserved. Therefore at zero temperature, in the absence of interactions, all atoms should be in the ground state with fluctuations vanishing. Hence, more restricted ensembles are better suited for the finite sample, trapped situation. The canonical fluctuations were first computed by Politzer [8]. We, on the other hand, 
devoted our attention to microcanonical ensemble. Its control parameters are the total energy of the system $E$ and the number of atoms $N$. The relevant partition function is the degeneracy of the $(E, N)$ subspace, which will be denoted by $\Gamma(N, E)$. The microcanonical partition function is related via power series expansion to the grand canonical one with the canonical partition function being an intermediate step

$$
\Xi(z, \xi)=\sum_{N=0}^{\infty} z^{N} Z(N, \xi)=\sum_{N=0}^{\infty} z^{N} \sum_{E=0}^{\infty} \xi^{E} \Gamma(N, E)
$$

Now, the microcanonical partition function in a natural way splits into a sum of degeneracy factors of the same total energy* $E$ but with different number of atoms in the ground state (or complementary number of atoms in all excited states):

$$
\Gamma(N, E)=\sum_{N_{\mathrm{ex}}=1}^{N} \Gamma_{\mathrm{ex}}\left(N_{\mathrm{ex}}, E\right) .
$$

The probability distribution, which carries all information about the statistics of the condensate, is

$$
P\left(N_{\mathrm{ex}}, E\right)=\frac{\Gamma_{\mathrm{ex}}\left(N_{\mathrm{ex}}, E\right)}{\Gamma(N, E)}
$$

Its moments may be computed via differentiation from a generating function

$$
Y(z, E)=\sum_{N_{\mathrm{ex}}=1}^{\infty} z^{N_{\mathrm{ex}}} \Gamma_{\mathrm{ex}}\left(N_{\mathrm{ex}}, E\right)
$$

In particular,

$$
N-\left\langle N_{0}\right\rangle=\left\langle N_{\mathrm{ex}}\right\rangle=\left.\left(z \frac{\partial}{\partial z}\right) Y(z, E)\right|_{z=1}
$$

and

$$
\delta^{2} N_{0}=\delta^{2} N_{\mathrm{ex}}=\left.\left(z \frac{\partial}{\partial z}\right)^{2} Y(z, E)\right|_{z=1} .
$$

The formula (3.5) has a form of a partition function of a new statistical ensemble [9]. Like the grand canonical one, it has an unspecified number of atoms while like the microcanonical one it has a well-defined energy. It describes the part of the system, which is excited. It is coupled to the condensate, serving as a reservoir of the particles, all at energy zero. We coined a term Maxwell demon ensemble for this situation. If the excited atoms were connected to a standard particle reservoir, a smart demon would have to be on guard letting only zero energy atoms in and out. As we see, summation in (3.5) has been extended to infinity. In our approximation we treat the condensate as infinitely large.

* Without loss of generality, in this paper we chose the origin of the energy scale at the energy of the ground state. 
One more step up to define the next generation function easily yields something familiar

$$
\sum_{E=1}^{\infty} \xi^{E} Y(z, E)=\Xi_{\mathrm{ex}}(z, \xi),
$$

the grand canonical partition function of the excited states, that is the product (3.3) with missing $n=0$ term. The expansion (3.8) is best achieved via the Cauchy integral

$$
Y(z, E)=\frac{1}{2 \pi \mathrm{i}} \oint \frac{\Xi_{\mathrm{ex}}(z, \xi)}{\xi^{E+1}} \mathrm{~d} \xi,
$$

where the integration contour runs around the origin of the complex $\xi$ plane. For $E \gg 1$ the steepest descent method may be used. All we need is asymptotic formula for $\Xi_{\text {ex }}$, or rather its logarithm. This may be easily found. In fact, the most detailed analysis of this function was given in [10]. Here we just need its leading high temperature term

$$
\ln \Xi_{\mathrm{ex}}(z, \xi)=-\frac{g_{4}(z \xi)}{(\ln \xi)^{3}},
$$

where the $g_{4}$ is the Bose function

$$
g_{n}(x)=\sum_{k=1}^{\infty} \frac{x^{k}}{k^{n}} .
$$

The rest is just the application of the saddle point approximation and the differentiation (3.6) and (3.7). The resulting expressions are giving the mean number of condensed atoms and its variance as a function of $N$ and $E$. To pass to a more conventional variable - the temperature - one may use either its microcanonical definition or, as a shortcut, just the expression known from the grand canonical formalism

$$
E=3 \zeta(4)\left(\frac{k T}{\hbar \omega}\right)^{4}
$$

where $\zeta$ denotes the Riemann zeta function. The results are

$$
\left\langle N_{0}\right\rangle=N\left[1-\frac{\zeta(3)}{N}\left(\frac{k T}{\hbar \omega}\right)^{3}\right],
$$

with the critical temperature - the one for which the mean number of condensed atoms becomes zero, and with the variance of this number equal to

$$
\delta^{2} N_{0}=\left(\frac{k T}{\hbar \omega}\right)^{3}\left[\zeta(2)-\frac{3 \zeta^{2}(3)}{4 \zeta(4)}\right] .
$$

The above formula holds (for large $N$ ) all the way to the critical temperature. Its value at the critical temperature gives the maximal fluctuations. As we see this is proportional to $N$. Therefore the variance itself is proportional to its square root. 
In statistical physics such fluctuations are called normal. Note that the variance, apart from its maximal value, does not depend on the number of atoms. This is the result of extending to infinity the sum in (3.5) or, more physically, the result of the condensate being macroscopically populated.

The finite sample results for the mean value are shown in Fig. 2, while such results for the variance are shown in Fig. 3. The exact data were obtained by means of the powerful, iterative numerical algorithm found by Gajda et al. [11].

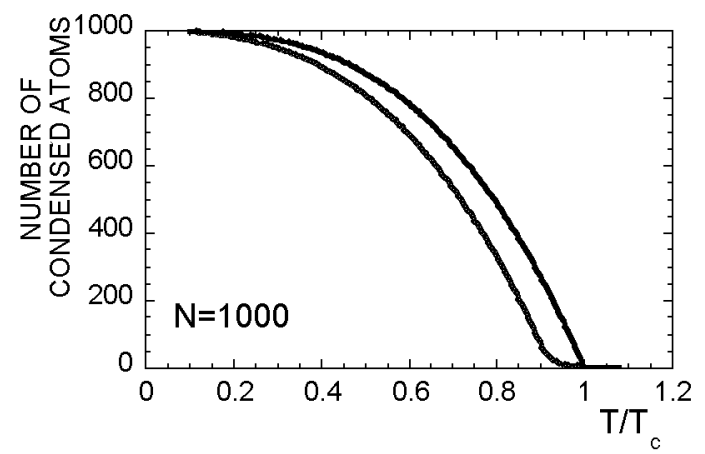

Fig. 2. Mean number of condensed atoms for the microcanonical ensemble of the ideal Bose gas in three-dimensional harmonic spherical trap (red). Blue curve is the asymptotic result.

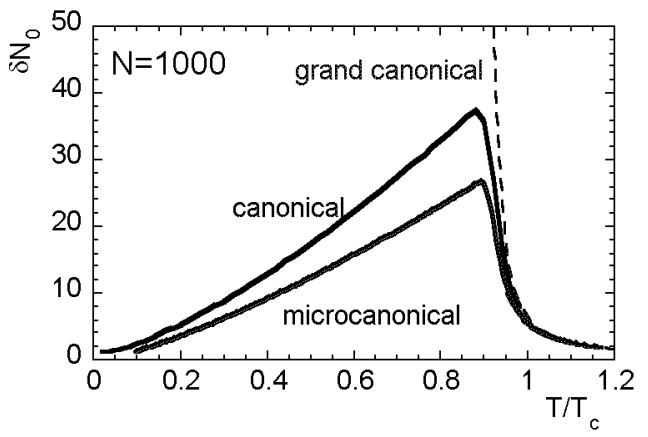

Fig. 3. Variance of the number of condensed atoms for the ideal Bose gas of 1000 atoms. Shown are results for three major statistical ensembles. Note non-physical character of the grand canonical ensemble fluctuations.

As we close this section let us mention an interesting link to the number theory. In one-dimensional harmonic oscillator, the microcanonical partition function is just a number of partitions of a given integer $E$ into a sum of $N$ summands. In fact, the well known generating function for this number of partitions is just (2.3) [12] and the gamma itself for not-too-large arguments is available in Mathematica.

The corrections to our fluctuations formula (3.14), which are due to weak interactions, were considered by several groups [13-16]. The result seems to depend 
on the assumptions made and, in our view, a problem remains unsolved. We come back to the role of interactions in Sec. 5 .

\section{How to observe fluctuations of the condensate?}

In the previous section we have described the microcanonical statistics of the ideal Bose gas confined in the harmonic potential. The experimental verification of these and other, competing predictions is scarce.

While the mean number of condensed atoms $N_{0}$ as a function of temperature has been measured in several laboratories [17], from the experimental point of view very little is known about fluctuations of BEC. The main source of information concerning higher-order correlations comes from the studies of the depletion of BEC due to inelastic two-body and three-body collisions [18]. This way the square and the cube of the population of condensed state were estimated. The experimental results have unequivocally ruled out the thermal fluctuations in the condensate implied by the grand canonical ensemble. Precision of those measurements is, however, unable to distinguish between sub-Poissonian and Poissonian fluctuations. The latter present in the coherent state, is often mentioned in connection with the Bogolyubov approximation [19].

In one of our recent papers [20] we have proposed to use a scattering of a short weak non-resonant laser pulses as a means of probing the BEC statistics. Before we shortly describe the method and the results, a remark concerning interactions is in order. Our study of the statistics of the ideal gas was based on enumeration of all possible configurations of the system which are compatible with the constraints imposed by two control parameters - the energy and the particle number. No experimenter can make a lot of condensates with identical $E$ and $N$. In realistic experiment, the shot to shot fluctuations of these parameters would inevitably mask the statistics we have computed.

What can be done instead, is a time series: interrogation of the system at equal time intervals and a build-up of the histogram of the frequencies, and a given occupation of the condensate is encountered. This method, on the one hand works only for the interacting gas (the ideal gas does not change) and on the other hand, needs the ergodic hypothesis (notoriously hard to verify) to be equivalent to the ensemble approach.

The proposed method of exploring the statistical properties of the condensate is based on the scattering of the series of short light pulses. The fluctuations in a condensate occur at the timescale given by the interatomic collisions, thus to probe the fluctuations the time delay between consecutive pulses should be of the order of milliseconds. The distribution of the number of photons scattered into a given solid angle should be measured. Out of the distribution of the number of scattered photons we may compute the mean and its variance. It is assumed that the pulse of light is weak and far detuned in order to avoid heating the condensate during the interaction. The pulse of light should be also sufficiently short in time. 
It should satisfy two conditions of technical nature. It should be so short that the atoms have no time to move far in the harmonic trap during the interaction. It should be also much shorter than the lifetime of the atomic transition to justify neglect of the spontaneous emission losses.

With these simplifications, one can first solve the Heisenberg equations satisfied by the atomic variables driven by the short pulse, and in the next stage, compute the scattered light field, treating the oscillating atomic dipoles as a given source of radiation. No back action of the radiated light on the motion of atomic dipoles is included. This is a neglect of spontaneous emission.

The resulting creation and annihilation operators of scattered photons are quadratic functionals of the atom field (the transition dipole moment is quadratic). As we have already mentioned, we need to compute the variance of number of scattered photons. This is quadratic in the photon operators, thus of the eighth order in atomic field. The computational complexity, both analytic and numerical is therefore significant. The results are summarized in Fig. 4. We have plotted the angular distribution of suitably normalized variance of the number of scattered photons. The calculations are performed for 1000 noninteracting atoms at the temperature of $0.3 T_{\mathrm{c}}$. We compare here the results for the grand canonical ensemble, designated as thermal, with the Poissonian statistics of the coherent state and with the microcanonical fluctuations. It is evident that the method proposed should easily discriminate not only between the non-physical thermal fluctuations and the rest but also between Poissonian and microcanonical predictions.

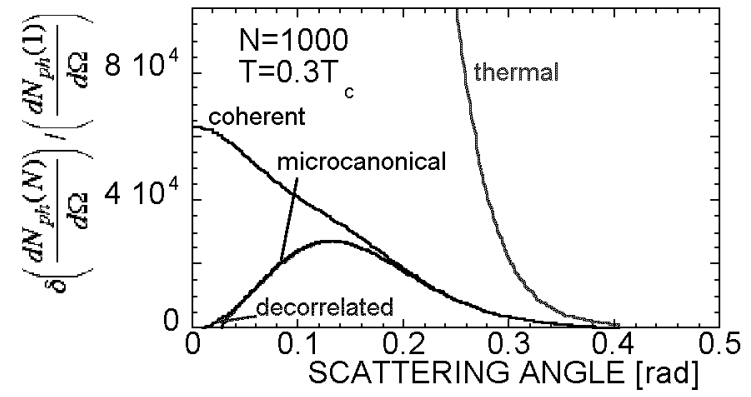

Fig. 4. Angular distribution of the variance of the number of scattered photons is shown. Note that not only the thermal but also the coherent states results are clearly distinguished. It is impossible, however, to distinguish between the microcanonical and the decorrelated results.

Regretfully, as indicated in the figure, microcanonical statistics is so narrow that it cannot be distinguished from nonfluctuating, Fock state results, called "decorrelated" in the figure. One would have to deal with even smaller condensates to reveal this difference.

The experiments probing the statistics remain a challenge. Perhaps the method outlined here will be of some help. In the next section we turn our 
attention to yet another aspect of the statistics - the spacial correlation functions.

\section{Coherences — a smoking gun for a Bose-Einstein condensation}

In previous sections we have shown that a Bose-Einstein condensate might be viewed as a giant matter wave of particles occupying the same quantum state. Such a picture cannot be automatically extended to an interacting system. The latter should be described by an $N$-body wave function (or density matrix) and single-particle states of a trapping potential do not have much of a physical significance. The criterion for the Bose condensation requires more detailed analysis. To a large extent, this goal has been completed many years ago by Penrose, Onsager, and Yang [21-23]. Let us imagine for a moment that an exact $N$-body wave function is given explicitly, and a problem in hand is, whether the system shows Bose-Einstein condensation. A conventional wisdom gives a hint: a picture of a giant matter wave should be still valid even in a case of interacting system. More precisely, a Bose-Einstein condensate should exhibit macroscopic quantum phenomena - phenomena that are inevitably related to large scale quantum correlation.

To illustrate basic concepts and subtlety of issue of a Bose-Einstein condensate we consider an exactly solvable model of particles trapped by an external harmonic potential and interacting by oscillatory forces [24] described by the following Hamiltonian:

$$
H=\frac{1}{2} \sum_{i=1}^{N}\left(p_{i}^{2}+x_{i}^{2}\right)+\frac{1}{2}\left(\frac{\omega^{2}-1}{N}\right) \sum_{i, j}\left(x_{i}-x_{j}\right)^{2},
$$

where $x_{i}$ is a position of the $i$-th particle and $p_{i}$ is its momentum. The parameter $\omega$ determines an interaction strength; $0<\omega<1$ corresponds to repulsive, while $\omega>1$ to attractive forces. All variables are expressed in units of harmonic potential of the trap. The assumed form of two-body interactions is not physical because of infinite range of two-body forces. However, from a point of view of criterion for a Bose condensation it might be quite useful. The ground state of the Hamiltonian in a simple case of $N=2$ is

$$
\Phi\left(x_{1}, x_{2}\right)=\left(\frac{1}{\pi}\right)^{3 / 4} \exp \left[-\frac{1}{4}\left(x_{1}+x_{2}\right)^{2}\right]\left(\frac{\omega}{\pi}\right)^{3 / 4} \exp \left[-\frac{\omega}{4}\left(x_{1}-x_{2}\right)^{2}\right] .
$$

The wave function Eq. (5.2) is a totally symmetric product of two terms: the center-of-mass one, which is a ground state of external trap, and the relative coordinate term which is a ground state of harmonic potential of frequency $\omega$. This form reveals a generic feature of any $N$-body interacting system. The center-of-mass experiences the external potential only. The relative degree of freedom wave function is translationally invariant. The two-particle eigenstate becomes a product of the 
ground state wave function of external potential, only if $\omega=1$. This signifies non-interacting case. A generalization of Eq. (5.2) to an arbitrary number of particles leads to the following expression for the ground state:

$$
\begin{gathered}
\Phi\left(\left\{x_{j}\right\}\right)=\left(\frac{1}{\pi}\right)^{d / 4} \exp \left(-\frac{1}{2} \boldsymbol{x}_{\mathrm{CM}}^{2}\right)\left(\frac{\omega}{\pi}\right)^{d(N-1) / 4} \\
\times \exp \left[-\frac{\omega}{2}\left(\sum \boldsymbol{\xi}_{j}^{2}-x_{\mathrm{CM}}^{2}\right)\right],
\end{gathered}
$$

where we used a short-hand notation $\left\{x_{i}\right\}=\left(x_{i}, \ldots, x_{N}\right)$, and

$$
x_{\mathrm{CM}}=(1 / \sqrt{N}) \sum_{1}^{N} x_{j}
$$

is an $N$-body center-of-mass coordinate (note an unconventional normalization). One might naively think that the ground state of a Bose system must be Bose condensed. We might, therefore, ask the question: which is this macroscopically occupied state?

To answer this question we should think for a moment about a possible detection and relate different measurements to quantum mechanical observables. Fortunately, in the early sixties (of the last century) Glauber discussed similar issue in detail [25]. He developed the coherence theory for a systematic description of the statistical properties of light. This is nothing else but higher-order coherences, which allow to distinguish a thermal from a laser light. Our task is in fact similar: we want to distinguish a classical system from a Bose-Einstein condensate. And we want to identify these experimentally observed features which are unique to a Bose condensate.

Needless to say, properties of a quantum system depend on a measurement performed. The simplest one consists of a numerously repeated detection of a single particle. According to quantum mechanics, the probability density of detecting a particle at $\boldsymbol{x}_{1}$ is equal to diagonal elements, $\boldsymbol{x}_{1}=\boldsymbol{y}_{1}$, of a one-particle reduced density matrix

$$
\rho^{(1)}\left(\boldsymbol{x}_{1} ; \boldsymbol{y}_{1}\right)=\operatorname{Tr}_{\{z\}}\left[\Phi^{*}\left(\boldsymbol{x}_{1}, z_{2}, \ldots, z_{N}\right) \Phi\left(\boldsymbol{y}_{1}, z_{2}, \ldots, z_{N}\right)\right] .
$$

One-particle measurement is, however, not the only detection, which can be performed on a many-body system. One can also simultaneously monitor a number of particles in a single shot of a camera. In such a case an observed particle configuration $\left\{x_{s}\right\}$ represents a single realization of the quantum system. This configuration is a statistical variable. It is distributed according to a diagonal part of $s$-particle reduced density matrix (high-order correlation function) $\rho^{(s)}\left(\left\{\boldsymbol{x}_{s}\right\} ;\left\{\boldsymbol{y}_{s}\right\}\right.$ ), defined analogously to (5.4). The joint detection can be related to a conditional measurement - a detection of a particle at some position $x_{s}$ provided that simultaneously other particles are detected at prescribed space-points $\left\{\boldsymbol{x}_{s-1}\right\}$. The relation 


$$
\begin{aligned}
\rho^{(s)}\left(\boldsymbol{x}_{s},\left\{\boldsymbol{x}_{s-1}\right\} ; \boldsymbol{y}_{s},\left\{\boldsymbol{x}_{s-1}\right\}\right) \\
\quad=\rho^{(1 / s-1)}\left(\boldsymbol{x}_{s} ; \boldsymbol{y}_{s} \mid\left\{\boldsymbol{x}_{s-1}\right\}\right) \rho^{(s-1)}\left(\left\{\boldsymbol{x}_{s-1}\right\} ;\left\{\boldsymbol{x}_{s-1}\right\}\right)
\end{aligned}
$$

defines conditional one-particle density matrix. Obviously, if particles are independent, then

$$
\rho^{(1 \mid s-1)}\left(\boldsymbol{x}_{s} ; \boldsymbol{y}_{s} \mid\left\{\boldsymbol{x}_{s-1}\right\}\right)=\rho^{(1)}\left(\boldsymbol{x}_{s} ; \boldsymbol{y}_{s}\right)
$$

and

$$
\rho^{(s)}\left(\left\{\boldsymbol{x}_{s}\right\} ;\left\{\boldsymbol{x}_{s}\right\}\right)=\prod_{j=1}^{s} \rho^{(1)}\left(\boldsymbol{x}_{j} ; \boldsymbol{x}_{j}\right) .
$$

Such a separation of $s$-order correlation function signifies the $s$-order coherence in the system. If the system is coherent, then joint and numerously repeated detection gives the same result. In the opposite case both detection schemes might give different answers. We will make use of this observation soon.

Being equipped with the basic knowledge of a detection process and the related correlation functions, we are ready now to analyze the model system. Due to a simple form of the wave function (5.3) all relevant reduced density matrices can be evaluated exactly. Moreover, they can be easily diagonalized, i.e. brought to the form

$$
\rho^{(1)}(\boldsymbol{x}, \boldsymbol{y})=\sum_{j} \lambda_{j} \varphi_{j}^{*}(\boldsymbol{x}) \varphi_{j}(\boldsymbol{y}) .
$$

A physical meaning of Eq. (5.7) is quite obvious. The one-particle subsystem is in a mixed state being a statistical mixture of one-particle "orbitals" $\varphi_{i}(\boldsymbol{x})$ with probabilities $\lambda_{i}$ being relative occupations of the $i$-th state. Diagonalization provides us with a natural and very convenient one-particle basis. In our case, in a limit of large $N$, a dominant eigenvalue is

$$
\lambda_{0}=\left(\frac{2}{1+\sqrt{1+N^{\kappa-1}+N^{-\kappa-1}}}\right)^{3 / 2},
$$

where we introduced an "interaction strength" parameter $\kappa$ related to the frequency $\omega$ by $\omega=N^{\kappa}$. Surprisingly, if $|\kappa|<1$, then $\lambda_{0} \approx 1$. One-particle subsystem is in a pure state even if every particle interacts with all remaining ones! From the viewpoint of one-particle measurements the system is undistinguishable from a system of non-interacting particles - all of them occupying the same state

$$
\varphi_{0}(\boldsymbol{x})=\left(\frac{\omega}{\pi}\right)^{3 / 2} \exp \left(-\frac{1}{2} x^{2}\right) .
$$

In such a situation the one-particle matrix separates

$$
\rho^{(1)}(x ; y) \approx \varphi_{0}^{*}(x) \varphi_{0}(y) .
$$

In Fig. 5 we plot relative population of the highest occupied state as a function of interaction strength $\kappa$ for different finite values of particle number $N$. Note a 




Fig. 5. Fraction of condensed atoms as a function of the interaction strength $\kappa$ for different number of particles; $N=10^{3}-$ green line; $N=10^{5}-$ red line; $N=10^{7}$ blue line.

symmetry with respect to a "sign" of interactions: $\kappa<0$ for repulsive, and $\kappa>0$ for attractive forces. The larger number of particles, the more rapidly dominant eigenvalue vanishes with increasing $|\kappa|$.

The model system is a perfect candidate for a Bose-Einstein condensate in a region of $|\kappa|<1$. Separability of one-particle density matrix justifies a picture of macroscopic occupation of one single-particle state. The system can be assigned a macroscopic wave function $\varphi_{0}(\boldsymbol{x})$ which furnishes a basis for the celebrated Gross-Pitaevskii equation and Bogolyubov approach [26]. Separability, in a language of the coherence theory, signifies the first-order coherence. Interference fringes with $100 \%$ visibility will be observed in a two-slit Young interference experiment performed on such a system. But this feature cannot be exclusively attributed to Bose condensates. Interference experiments have been successfully performed also with thermal, but sufficiently monochromatic, atomic beams [27]. If the first-order coherence were the only quantum feature of Bose condensates, they would be really poor condensates. Genuine condensates, similarly as genuine lasers, ought to show higher-order coherences. In the case studied here it can be shown $[28,29]$ that in the region of $|\kappa|<1$ the system shows also higher-order coherences,

$$
\rho^{(s)}\left(\left\{x_{s}\right\} ;\left\{y_{s}\right\}\right)=\prod_{i=1}^{s} \rho^{(1)}\left(x_{i} ; \boldsymbol{y}_{i}\right),
$$

provided that $s \ll N$. This inequality is an important limitation. The coherent state of the electromagnetic field is coherent in all orders.

The studied example teaches us another important lesson. A ground state of interacting Bose system is not necessarily a Bose-Einstein condensate. At least if the first-order coherence is considered. If one performs a Young experiment and collects data from numerously repeated one-particle detection, then no interference will be observed. A reason for this decoherence originates in existence of two drastically different length scales. Amplitude of a zero point oscillations 
of the center-of-mass is fixed because is related to the external potential. On the contrary, spatial spreading of relative coordinates depends on the interaction strength and particle number. For strong repulsive forces it exceeds significantly the center-of-mass spreading. In a case of attractive forces the system is aggregated over a distance much smaller than quantum uncertainty of the center-of-mass. A spatial extension of the system revealed in a one-particle measurement is related to larger of the two length scales. But the first-order coherence length is limited to smaller of them. That is why the system does not show coherences on the macroscopic scale comparable to its size.

Can one, however reveal some coherence in a joint detection of a large number of particles? Let us consider the simplest case of a conditional measurement - position of a particle is collected only when simultaneously another one is being observed at a given location $\boldsymbol{x}_{0}$. Corresponding reduced density matrix can be evaluated in the model. Its diagonal part is a probability density of conditional detection and it is shown in Fig. 6 for a case of attractive interactions $\kappa=1.3$ (blue line). For a comparison the red line shows one-particle probability density

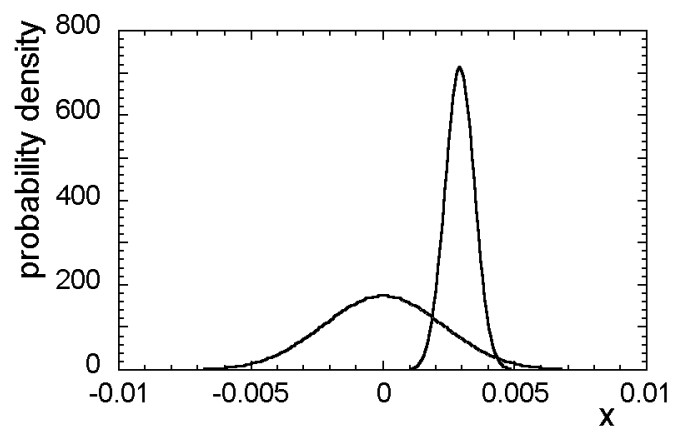

Fig. 6. One-particle probability density (in dimensionless units); single-particle detection - red line; conditional detection of a particle provided that simultaneously another one have been found at $x_{0}=0.003$ - blue line. Total number of particles $N=10^{4}$, and interaction strength $\kappa=13$.

$\rho^{(1)}(\boldsymbol{x}, \boldsymbol{x})$ which is spread widely over a large distance. This spatial extension defines a region where the first particle can be effectively detected. We assumed that the particle has been found at $x_{0}=0.003$. As the first particle is detected at some point, the second one must be (at the same time) relatively close. Because of a strong attraction, particles are correlated. Conditional detection is a practical realization of a measurement performed in the center-of-mass frame in a case when position of the latter has large quantum uncertainty. The center-of-mass length scale is eliminated in a single realization of a quantum system. What remains it is only a relative degree of freedom spreading which determines a distance on which the system shows the coherence. Analysis of eigenvalues of corresponding conditional density matrix supports this interpretation. There exists single dominant 
eigenvalue equal (in large $N$ limit) to

$$
\lambda_{0}=\left(\frac{2}{1+\sqrt{2}}\right)^{3 / 2}
$$

Such system is a Bose-Einstein condensate despite that it cannot be assigned a macroscopic wave function.

Situation is completely different if particles repel each other. The joint measurement does not reveal any coherences if $\kappa<-1$. The larger length scale is common to all $N-1$ relative degrees of freedom and cannot be eliminated by a joint measurement. In our model, strong repulsive two-body interactions lead to inevitable destruction of the Bose-Einstein condensate even in the ground state of a bosonic system.

\section{Interacting Bose-Einstein condensate at finite temperatures}

Theoretical description of a stationary Bose-Einstein condensate is a quite complicated and subtle problem even at zero temperature. One can easily imagine, therefore, that a study of a dynamical behavior of the system, in particular at finite temperatures, is a real challenge. The best suited language for that kind of studies is a language of field theory. $N$-particle Bose system is being assigned a bosonic field operator $\hat{\Psi}(x)$ that destroys a particle at position $x$ and obeys standard bosonic commutation relations with its conjugated partner $\hat{\Psi}^{+}(\boldsymbol{x})$ creating a particle at $x$ :

$$
\left[\hat{\Psi}(x), \hat{\Psi}^{+}(y)\right]=\delta^{3}(x-y) .
$$

$N$-particle Hamiltonian can be expressed in terms of the field operator

$$
H=\int \hat{\Psi}^{+}(x) H_{0}(x) \hat{\Psi}(\boldsymbol{x})+\frac{1}{2} \iint \hat{\Psi}^{+}(x) \hat{\Psi}^{+}(\boldsymbol{y}) U(\boldsymbol{x}-y) \hat{\Psi}(\boldsymbol{x}) \hat{\Psi}(\boldsymbol{y})
$$

where $H_{0}(\boldsymbol{x})$ is a single particle Hamiltonian while $U(\boldsymbol{x}-\boldsymbol{y})$ is a two-body interaction potential. In realistic situations of Bose condensates of dilute atomic vapors in magnetic traps the two-body interaction is approximated by a contact potential

$$
U(x-y)=g \delta^{3}(x-y),
$$

where $g$ characterizes the atom-atom interaction in the low-energy, $s$-wave approximation. A Heisenberg equation originating from the Hamiltonian (6.2) acquires a form

$$
\mathrm{i} \hbar \frac{\partial}{\partial t} \hat{\Psi}=H_{0} \hat{\Psi}+g \hat{\Psi}^{+} \hat{\Psi} \hat{\Psi}
$$

A full operator solution of (6.4) is impossible. There are different approaches to a time evolution of an interacting condensate. They lead to numerical algorithms, which are hard to implement $[30,31]$. None of them are in fact capable to handle a realistic case of a large number of particles at a relatively high temperature. But this is only the first obstacle which one encounters while studying an interacting 
Bose system at finite temperatures. It is not easy to extract relevant physical information like, for example, a mean occupation and fluctuations of a condensate - even having obtained the field operator. It obviously requires an unique identification of a condensed part of the system. We have already discussed this delicate issue in the previous section. A genuine condensate can be assigned a condensate wave function which is an eigenvector corresponding to a dominant eigenvalue of a one-particle density matrix. This matrix can be expressed with the help of field operators

$$
\left\langle\hat{\Psi}^{+}(x) \Psi(y)\right\rangle=N \rho^{(1)}(x ; y),
$$

and the average is taken in the initial state of many-body system. In a general case, a diagonalization of a density matrix is needed in order to determine physically relevant quantities. There is however, one particular situation when symmetry of a problem is of great help. This is the case of a gas trapped in a box with periodic boundary conditions. The symmetry of the confining potential uniquely enforces a form of eigenstates of the one-particle density matrix regardless of interaction. The density matrix must be periodic and depend only on a difference of its arguments

$$
\rho^{(1)}(x ; y)=\rho^{(1)}(x-y)=\sum \lambda_{k} f_{k}^{*}(x) f_{k}(y)
$$

The above condition indicates that eigenfunctions are simply plane waves

$$
f_{k}(x)=\frac{1}{L^{3 / 2}} \exp (-\mathrm{i} k \cdot x)
$$

and the wave vector $\boldsymbol{k}$ takes quantized values $\boldsymbol{k}=(2 \pi / L) n$ with $n_{i}=0, \pm 1, \pm 2, \ldots$ ( $i=x, y, z$ ), and $L$ being a length of the cubic box. A stationary state of a Bose-Einstein condensate corresponds, therefore, to $k=\mathbf{0}$ state of the box. This is the reason why quite often a Bose condensation is being thought of as a condensation in a momentum space. If we expand the field operator in the basis of a box eigenfunctions

$$
\hat{\Psi}(x)=\sum f_{k}(x) \hat{a}_{k},
$$

then we save the effort of diagonalization a density matrix, simultaneously gaining a direct insight into physical quantities. The operator $\hat{a}_{\boldsymbol{k}}\left(\hat{a}_{\boldsymbol{k}}^{+}\right)$annihilates (creates) a particle in the state $f_{\boldsymbol{k}}(\boldsymbol{x})$, and $\hat{n}_{\boldsymbol{k}}=\hat{a}_{\boldsymbol{k}}^{+} \hat{a}_{\boldsymbol{k}}$ counts particles occupying this state. Using an advantage of the periodic symmetry we limit our study to a condensate trapped in a periodic box. In this case a one-particle Hamiltonian is

$$
H_{0}=\frac{p^{2}}{2 m},
$$

and dynamical equation for the operators $a_{k}$ (in the interaction picture) acquire the following form:

$$
\hat{a}_{\boldsymbol{k}}=-\mathrm{i} g \sum \exp \left[2 \mathrm{i}\left(n-n_{1}\right)\left(n-n_{2}\right) t\right] \hat{a}_{\boldsymbol{k}_{1}+\boldsymbol{k}_{2}-\boldsymbol{k}}^{+} \hat{a}_{\boldsymbol{k}_{1}} a_{\boldsymbol{k}_{2}} .
$$

The two-body interaction energy $g$ and time $t$ are expressed in units of $\Delta=$ $\frac{\hbar^{2}}{2 m}\left(\frac{\frac{2}{\pi}}{L}\right)^{2}$ and $\tau=\hbar / \Delta$ respectively. Obviously, some approximations are necessary if one wants to solve the nonlinear operator equations (6.8). A semiclassical 
approximation introduced in [32, 33] is particularly well suited for a description of the system at temperatures below the critical one, except the region close to the absolute zero. The approximation consists in replacing all creation and annihilation operators by $c$-numbers $\hat{a}_{\boldsymbol{k}} \rightarrow a_{\boldsymbol{k}}$. Such replacement is legitimate if a population of a given mode is greater than its quantum fluctuations, i.e. if $n_{k}=a_{k}^{*} a_{\boldsymbol{k}}>1$. At temperatures close to zero it is only $\boldsymbol{k}=\mathbf{0}$ mode for which the latter condition is fulfilled. Quantum fluctuations in excited modes are important. At higher temperatures, however, many modes are macroscopically populated and then thermal fluctuations dominate quantum ones.

The semiclassical approximation leads to a set of coupled nonlinear equations, which have to be solved numerically. Dynamical equations (6.8) can be viewed as equations for coupled macroscopically occupied "mean fields" $a_{\boldsymbol{k}}$. Our approximation is an extension of a Bogolyubov approach [26]. This approach is valid at low temperatures because it assumes that only $\boldsymbol{k}=\mathbf{0}$ mode is macroscopically occupied and therefore only the "condensed" mode, described by a complex field, is taken into consideration. Evidently, for finite system at higher temperatures other modes can be treated in the same fashion. Let us observe that our approximate dynamics conserves both the total energy and the number of particles. It corresponds therefore, to a genuine microcanonical description.

Our first goal is to describe the equilibrium state of an interacting system. We assume that our dynamics reaches a steady state for any "typical" initial condition. This assumption is very important and should be carefully tested [33, 34]. One-dimensional version of this dynamics is completely integrable [35], however, in higher number of dimensions or in a case of more realistic trapping potentials, the evolution may be chaotic. We start the evolution choosing the initial "Bose-Einstein-like" distribution of populations $\left|a_{k}\right|^{2}$. In determining this distribution, we neglect all interactions, thus the initial condition evidently does not correspond to the equilibrium distribution of the interacting system. Moreover, there is still a freedom in selecting phases of the coupled fields. In our approach, each mode is assigned an initial, randomly chosen, phase. Any subsequent dynamics depends on initial phases and, in a sense, describes a single realization of the quantum system. Microcanonical expectation values would require the average over the initial phases. Instead of doing so, we trace the system for a sufficiently long time and compute time averages. In fact, time averages rather than ensemble ones are measured experimentally. In addition, the two are equal if a system is ergodic.

In our three-dimensional calculations we assumed that $N=100000$ rubidium $\left({ }^{87} \mathrm{Rb}\right)$ atoms are trapped into a cubic box of a size equal to the Thomas-Fermi radius of the same system trapped in a harmonic trap of frequency $2 \pi \times 80 \mathrm{~Hz}$. The two-body interaction strength is $g / \Delta=2.52 \times 10^{-4}$. We performed our calculations with 729 modes. Further increase in a number of modes does not lead to any substantial modifications of final results. We traced the evolution of the system on 
a timescale equal approximately to one second. The calculations show that after few milliseconds the system reaches a state of a dynamical equilibrium regardless the choice of initial phases. The occupation of the $k=\mathbf{0}$ mode stabilizes at some value, and on a larger timescale it only fluctuates around that value, Fig. 7 .

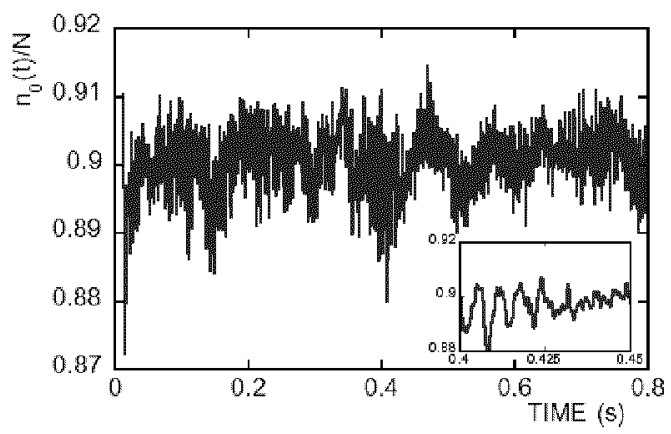

Fig. 7. Condensate occupation as a function of time for total energy per particle $E / \hbar=$ $539 \mathrm{~Hz}$.

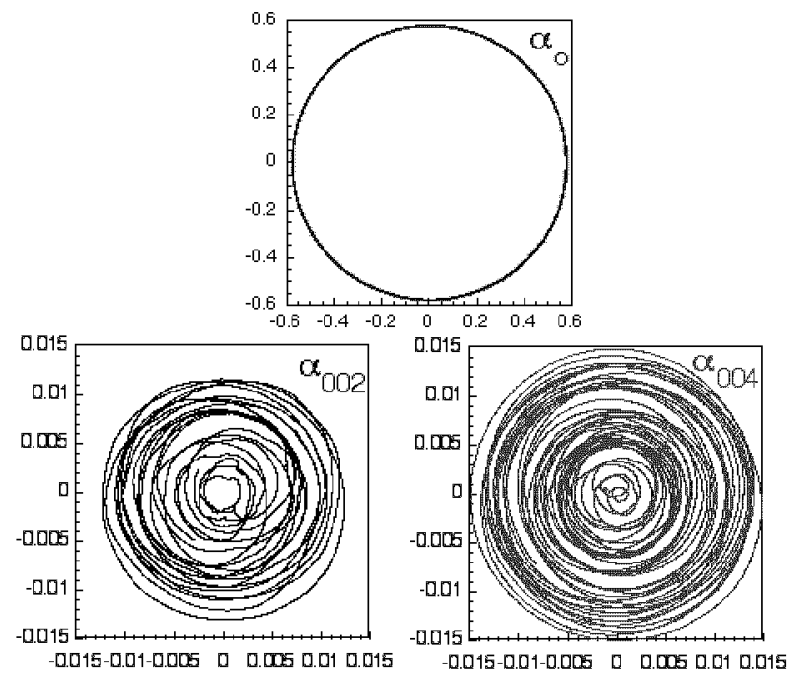

Fig. 8. Phase-space portraits - imaginary versus real part of complex amplitudes corresponding to different modes of the system.

In Fig. 8 we present a typical "phase-space portraits" of some complex amplitudes $a_{k}$. The figure depicts imaginary versus real part of amplitudes corresponding to $k=(0,0,0), k=(0,0,2)$, and to $k=(0,0,4)$ modes. Different points correspond to different instances of time. Two important features could be observed. First of all, we stress a striking difference between $k=\mathbf{0}$ mode (a condensate) and higher modes. The amplitude of condensate mode acquires a steady-state value and its relative fluctuations are small. On the contrary, relative fluctuations of 
higher modes reach $100 \%$. The second observation is that a phases of all complex fields $a_{k}$ varies uniformly over the whole accessible range between zero and $2 \pi$. Figure 8 provides a strong indication that the dynamics studied here is ergodic and the system visits all accessible states which are allowed by conservation of energy and particle number.

In Fig. 9 a mean population (blue line) of the interacting Bose-Einstein condensate and its fluctuations (red line) are presented as a function of the total energy of the system. Both curves are smooth and do not show any singularity characteristic of a phase transition. This behavior is not surprising because our system is finite. We observe that a condensate population decreases with the energy and finally disappears at the energy close to $E / \hbar=2000 \mathrm{~Hz}$. The fluctuations grow with energy and reach their maximal value at $E / \hbar=1450 \mathrm{~Hz}$. Let us observe that it is also an inflection point of a mean condensate population. In a vicinity of that energy the system undergoes rapid changes. This energy is a Bose-Einstein condensation energy.

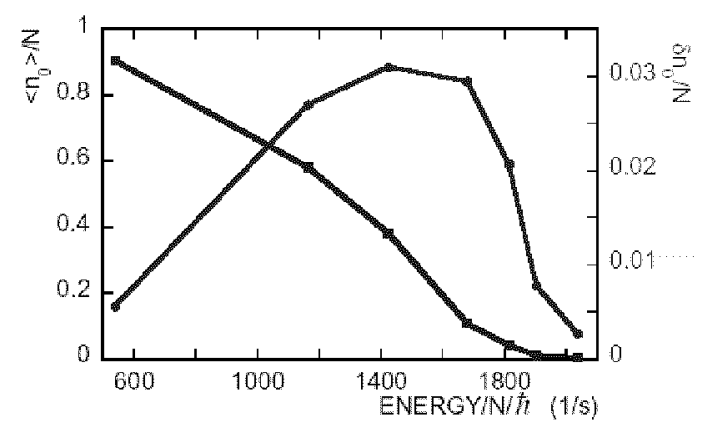

Fig. 9. Condensate occupation (in blue) and fluctuations (in red) versus total energy per particle.

The method presented here is very powerful. A careful reader, however, might be confused. By replacing creation and annihilation operators by $c$-numbers $\hat{a}_{\boldsymbol{k}}^{+}, \hat{a}_{k} \rightarrow a_{k}^{*}, a_{\boldsymbol{k}}$ we simultaneously substituted the field operator by a "classical wave function"

$$
\hat{\Psi}(x) \rightarrow \sqrt{N} \Psi(x)=\sum_{k} a_{k} f_{k}(x),
$$

and the set of equations (6.8) is in fact nothing else but the celebrated Gross-Pitaevskii equation written in the basis of plane waves. This equation traditionally describes a pure condensate at zero temperature. The corresponding one-particle density matrix

$$
\rho^{(1)}(\boldsymbol{x} ; \boldsymbol{y})=\Psi^{*}(\boldsymbol{x}) \Psi(\boldsymbol{y}),
$$

projects onto a pure state $\Psi(x)$ which is macroscopically occupied by $N$ atoms. What about our arguments that it is $\left|\boldsymbol{\alpha}_{0}\right|^{2}$ which gives population of a condensate? 
Have we been cheating so far? Certainly not! We owe, however, an explanation. And again, the detailed analysis of a detection process helps to resolve the issue.

We admit that in fact we are solving the Gross-Pitaevskii equation. But the initial state is far from equilibrium. The system, therefore, is strongly developing, and the wave function $\Psi(x)$ varies rapidly in time. Complex amplitudes of different modes oscillate very fast, even when the evolution reaches a stage of dynamical equilibrium. Needless to say, a detection process is not instantaneous. Optical methods are used to monitor an atomic Bose-Einstein condensate - a CCD camera monitors a light passing through a condensate. An exposition time, $\tau$, varies from few microseconds up to several hundreds of microseconds thus what is being observed it is a time average of a diagonal part of a one-particle reduced density matrix

$$
\left\langle\rho^{(1)}(\boldsymbol{x}, \boldsymbol{y}, t)\right\rangle_{t}=\left\langle\Psi^{*}(\boldsymbol{x}, t) \Psi(\boldsymbol{y}, t)\right\rangle_{t}=\frac{1}{N} \sum_{\boldsymbol{k}, \boldsymbol{k}^{\prime}}\left\langle a_{\boldsymbol{k}}^{*}(t) a_{\boldsymbol{k}^{\prime}}(t)\right\rangle_{t} f_{\boldsymbol{k}}^{*}(\boldsymbol{x}) f_{\boldsymbol{k}^{\prime}}(\boldsymbol{y})
$$

This time a verage "spoils" a purity of dynamically evolving state. The detection introduces a coarse-grained time. Rapidly oscillating phases of different mean fields average to zero on that timescale, and only diagonal elements survive

$$
\left\langle a_{\boldsymbol{k}}^{*}(t) a_{\boldsymbol{k}^{\prime}}(t)\right\rangle_{t}=\delta_{\boldsymbol{k}, \boldsymbol{k}^{\prime}}\left\langle n_{\boldsymbol{k}}(t)\right\rangle
$$

Moreover, if the system reaches a state of dynamical equilibrium, averaged occupations of different modes do not depend on time (they depend only on the total energy and the number of particles) and one-particle density matrix acquires a stationary form

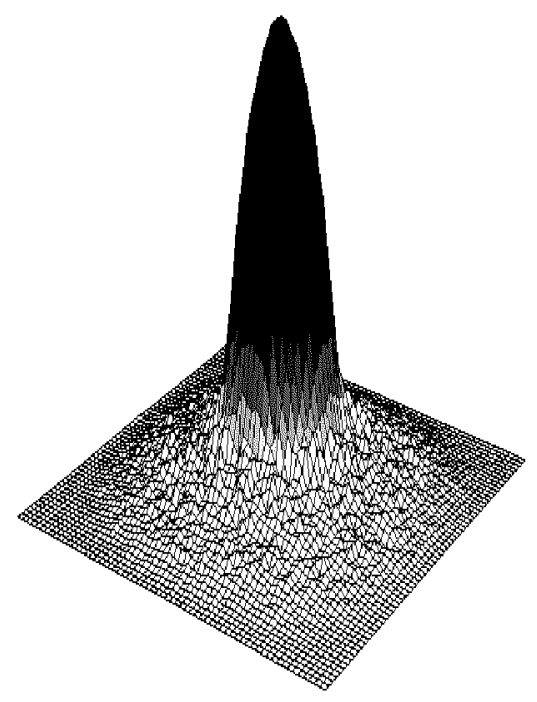

Fig. 10. Density of an interacting Bose gas at finite temperature in a harmonic trap. 


$$
\left\langle\rho^{(1)}(\boldsymbol{x} ; \boldsymbol{y})\right\rangle_{t}=\sum_{\boldsymbol{k}} \frac{\left\langle n_{k}\right\rangle_{t}}{N} f_{\boldsymbol{k}}^{*}(\boldsymbol{x}) f_{\boldsymbol{k}}(\boldsymbol{y}) .
$$

Equation (6.13) furnishes a basis for our method.

It is obvious that the same method can be used in a more realistic case of a condensate trapped in a harmonic potential. By averaging the product of a time-dependent solution of the Gross-Pitaevskii equation $\Psi^{*}(\boldsymbol{x}, t) \Psi(\boldsymbol{y}, t)$ over a finite observation time we obtain the one-particle density matrix. In Fig. 10 the one-particle density of interacting Bose gas trapped in magnetic trap is shown. One can easily see a broad thermal cloud and a sharp peak of the Bose condensed atoms on top of it. Do not confuse the figure with an experimental result. This is the theoretical figure obtained within our approach! It strongly resembles the results of real experiments. And, as it is done in experiments, by identifying the two components (the condensate and the thermal cloud) it is possible to determine a condensate population and a temperature. We are currently working on the application of the above method to the quantitative analysis of the properties of the ultracold Bose gas, trapped in the harmonic potential just below the critical temperature [36].

\section{Acknowledgments}

The results presented here were obtained in collaboration with J. Mostowski, M. Załuska-Kotur, Z. Idziaszek, and K. Góral. M.G. thanks the State Committee for Scientific Research, grant 2P03B07819, for support, while the research of K.R. was sponsored by the subsidy from the Foundation for Polish Science.

\section{References}

[1] S.N. Bose, Z. Phys. 26, 178 (1924).

[2] A. Einstein, Sitzber. Kgl. Preuss. Akad. Wiss. 22, 261 (1924).

[3] S. Chu, Rev. Mod. Phys. 70, 685 (1998); C. Cohen-Tannoudji, Rev. Mod. Phys. 70, 707 (1998); W.D. Phillips, Rev. Mod. Phys. 70, 721 (1998).

[4] M.H. Anderson, J.H. Ensher, M.R. Mattews, C.E. Wieman, E.A. Cornell, Science 269, 198 (1995).

[5] K.B. Davies, M.-O. Mewes, M.R. Andrews, N.J. van Druten, D.S. Durfee, D.M. Kurn, W. Ketterle, Phys. Rev. Lett. 75, 3969 (1995).

[6] K. Huang, Statistical Mechanics, Wiley, New York 1963.

[7] E. Schrödinger, Statistical Thermodynamics, Dover Publ., New York 1989; I. Fujiwara, D. ter Haar, H. Wergeland, J. Stat. Phys. 2, 329 (1970).

[8] H.D. Politzer, Phys. Rev. A 54, 5048 (1996).

[9] P. Navez, D. Bitouk, M. Gajda, Z. Idziaszek, K. Rzążewski, Phys. Rev. Lett. 79, 1789 (1997).

[10] M. Gajda, K. Rzążewski, Phys. Rev. Lett. 78, 2686 (1997).

[11] M. Gajda, Z. Idziaszek, K. Rzążewski, P. Navez, Mol. Phys. Rep. Supplement 1, 46 (2001). 
[12] M. Abramovitz, I.A. Stegun, Handbook of Mathematical Functions, Dover Publ., New York 1970.

[13] S. Giorgini, L.P. Pitaevskii, S. Stringari, Phys. Rev. Lett. 80, 5040 (1998).

[14] Z. Idziaszek, M. Gajda, P. Navez, M. Wilkens, K. Rzążewski, Phys. Rev. Lett. 82, 4376 (1999).

[15] F. Meier, W. Zwerger, Phys. Rev. A 60, 5133 (1999).

[16] V.V. Kocharovsky, V.V. Kocharovsky, M.O. Scully, Phys. Rev. Lett. 84, 2306 (2000).

[17] J. R. Enscher, D.S. Jin, M.R. Matthews, C.E. Wieman, E.A. Cornell, Phys. Rev. Lett. 77, 4984 (1996); M.-O. Mewes, M.R. Andrews, N.J. van Druten, D.M. Kurn, D.S. Durfee, W. Ketterle, ibid. 77, 416 (1996).

[18] E.A. Burt, R.W. Ghrist, C.J. Myatt, M.J. Holland, E.A. Cornell, C.E. Wieman, Phys. Rev. Lett. 79, 337 (1997); D.M. Stamper-Kurn, H.-J. Miesner, S. Inouye, M.R. Andrews, W. Ketterle, ibid. 81, 500 (1998); similar methods have been used to detect the phase transition in 2D spin polarized hydrogen gas: A.I. Safonov, S.A. Vasilyev, I.S. Yasnikov, I. Lukashevich, S. Jaakkola, ibid. 81, 4545 (1998).

[19] N.N. Bogolyubov, J. Phys. (Moscow) 11, 231 (1947).

[20] Z. Idziaszek, K. Rzążewski, M. Lewenstein, Phys. Rev. A 61, 053608 (2000).

[21] O. Penrose, Philos. Mag. 42, 1373 (1951).

[22] O. Penrose, L. Onsager, Phys. Rev. Lett. 104, 576 (1956).

[23] C.N. Yang, Rev. Mod. Phys. 34, 694 (1962).

[24] M. Załuska-Kotur, M. Gajda, A. Orłowski, J. Mostowski, Phys. Rev. A 61, 033631 (2000)

[25] R.J. Glauber, in: Quantum Optics and Electronics, Eds. C. De Witt, A. Blandin, C. Cohen-Tannoudji, Gordon and Breach, New York 1965, p. 63; Phys. Rev. 130, 2529 (1963); Phys. Rev. 131, 2766 (1963).

[26] F. Dalfovo, S. Giorgini, L.P. Pitaevskii, S. Stringari, Rev. Mod. Phys. 71, 463 (1999); A.J. Leggett, Rev. Mod. Phys. 73, 307 (2001).

[27] For an overview of atom interferometry see Atom Interferometry, Ed. P. Berman, Academic, San Diego 1997, and references therein.

[28] M. Gajda, M. Załuska-Kotur, J. Mostowski, J. Phys. B, At. Mol. Opt. Phys. 71, $4003(2000)$.

[29] M. Gajda, M. Załuska-Kotur, J. Mostowski, Opt. Express 8, 106 (2000).

[30] C.W. Gardiner, P. Zoller, Phys. Rev. A 55, 2902 (1997); D. Jaksch, C.W. Gardiner, P. Zoller, Phys. Rev. A 56, 575 (1997).

[31] R. Walser, J. Williams, J. Cooper, M. Holland, Phys. Rev. A 59, 3878 (1999).

[32] K. Góral, M. Gajda, K. Rzążewski, Phys. Rev. Lett. 86, 1397 (2001).

[33] K. Góral, M. Gajda, K. Rzążewski, Opt. Express 8, 92 (2000).

[34] M.J. Davis, S.A. Morgan, K. Burnett, Phys. Rev. Lett. 87, 160402 (2001).

[35] F.M. Izrailev, B.V. Chirikov, Dokl. Akad. Nauk SSSR 166, 57 (1966) [Sov. Phys. Dokl. 11, 30 (1966)].

[36] K. Góral, M. Gajda, K. Rzążewski, in preparation. 